\title{
Modeling Tumor Growth for Kidney Cancer Based on Nuclei Clusters of Pathology Slides
}

\author{
Aydin Saribudak, Yiyu Dong, James Hsieh, and M. Umit Uyar
}

\begin{abstract}
Descriptive features of nuclei positions from tumor tissue samples are studied to construct mathematical models for tumor growth. Extracted from kidney cancer patients, tumorous tissue pieces are implanted in the flanks of mice to measure the course of tumor mass, which are then sampled on glass slides. H\&E slides are digitized under light microscope and analyzed to identify the structure of nuclei positions. Using $k$-means clustering method, the nuclei locations of each $\mathrm{H} \& \mathrm{E}$ slide are evaluated. The cluster features are used as inputs to our artificial intelligence based personalized tumor growth parameter computation method, called PReP-C. The exponential linear tumor growth model parameters and the corresponding growth curves computed by $P R e P-C$ are compared to the preclinical tumor volume measurements. The correlation between the computed results and the measurements from $14 \mathrm{H \& E}$ pathology slides is encouraging to build personalized mathematical models for tumor growth.
\end{abstract}

Index Terms-H\&E slide, tumor growth models, kidney cancer, exponential linear model, k-means clustering.

\section{INTRODUCTION}

Modeling tumorous cell proliferation has an effective role in cancer research to study the characteristics of disease progress. Empirical models describe tumor growth using mathematical equations without in-depth analysis of basal processes, whereas functional models concentrate on physiological hypotheses and biochemical processes [1], [2]. Exponential-linear tumor growth model [1] built based on experimental observations is a well-accepted empirical model. Tumor mass grows exponentially until it reaches a certain weight and volume, at which increase in tumor size becomes linear due to nutritional and oxygen limitations [3]. In exponential-linear model, tumor growth is expressed as a function of rate constants and tumor weight or volume when no drug is administered [1]. In our previous work [4], we computed tumor growth curves using genetic information from breast cancer patients in our artificial intelligence based methods. In this paper, we study the generation of tumor

Manuscript received January 19, 2015; revised April 20, 2015

A. Saribudak and M. U. Uyar are with Dept. of Electrical Eng., The City College of the City University of New York, USA (e-mail: \{asaribudak,uyar\}@ccny.cuny.edu).

Y. Dong and J. Hsieh are with Memorial Sloan Kettering Cancer Center, New York, USA (e-mail: \{dongy,hsiehj\}@mskcc.org). A. Saribudak and Y. Dong are co-first authors.

The initial research used in this work was supported by U.S. Army Communications-Electronics RD\&E Center contracts W15P7T-09-CS021 and W15P7T-06-C-P217, and by the National Science Foundation grants ECCS-0421159, CNS-0619577 and IIP-1265265. The contents of this document represent the views of the authors and are not necessarily the official views of, or are endorsed by, the U.S. Government, Department of Defense, and Department of the Army or the U.S. Army Communications Electronics RD\&E Center. growth models based on stained tissue samples of pathology slides from kidney cancer patients.

Hematoxylin-Eosin (H\&E) staining is a technique to visualize tumorous tissue components dyed with a number of stains of different colors [5]. Under a microscope a pathologist identifies cell nuclei stained in blue color by hematoxylin and cytoplasm and connective tissues stained in pink color by eosin [5]. The particles distributed on H\&E slides are clinically examined by a pathologist to determine the grade and the type of cancer. We analyze the spatial distribution of nuclei on H\&E pathology slides and nuclei clusters to identify the patient specific features.

Clustering is a method to group data sets, where each cluster consists of similar data points [6]. Structure of clusters is quantified by a variety of methods reported in literature [7], [8], [9]. Typically, the validity of clusters is evaluated by either the dispersion of data each cluster contains, or the data separation between clusters, or both [8]. We apply k-means clustering approach to $\mathrm{H} \& \mathrm{E}$ pathology slides to analyze the spatial distribution of nuclei.

In our process, we have first obtained human kidney tumor samples from kidney cancer patients. The samples are cut into small fragments and implanted in the flanks of mice in Memorial Sloan Kettering Cancer Center (MSKCC) to measure tumor volume for approximately one-month period The tissue samples are then collected and equipped on glass slides for H\&E staining. Digitized H\&E slide samples are analyzed to examine the relation between nuclei distribution specific to each slide and the tumor growth characteristics. Using $k$-means clustering method we define the clusters of nuclei on H\&E slide samples and determine the features. At the end of the data extraction process, we use the features from each H\&E slides in our artificial intelligence based Personalized Relevance Parameterization (PReP-C) approach to compute patient specific tumor growth curves. In this study, the correlation between the computed results by PReP-C and the measurements from $14 \mathrm{H} \& \mathrm{E}$ pathology slides is encouraging.

\section{MOdeling TUMOR GROWTH}

Exponential-linear model [1], [10], [11] is an empirical method to analyze the tumor cell proliferation quantitatively based on parameters defined for exponential and linear phases of tumor growth. We build artificial intelligence based mathematical models to compute tumor growth and related parameters including exponential-linear model parameters. In our earlier research [4], we have studied the relationship between the genetic information from breast cancer patients and the growth parameters, based on the genetic data retrieved from 79 breast cancer patients with ER+ status 
provided in I-SPY 1 TRIAL database [12]. Using the expression values of 74 breast cancer related genes from these patients and the tumor volume measurements obtained from NBIA database [13] as inputs to our computational models, we have computed tumor growth parameters. In this paper, building on our earlier results, we utilize H\&E slides of kidney cancer patients as the patient specific data source to model tumor proliferation for computations in $P \operatorname{ReP}-\mathrm{C}$ model.

Based on the extracted features of nuclei clusters, we construct an $\mathrm{m}$ dimensional feature data vector, namely $\boldsymbol{C}_{p_{x}}$, for a patient $p_{x}$. In vector $\boldsymbol{C}_{p_{x}}$, the correlation between features is expressed mathematically with the parameter assignment function $\zeta_{\mathrm{i}}$ which is stated as:

$$
\zeta_{i}=\prod_{j=1}^{\left|S_{i}\right|} v_{i, j}
$$

where $v_{i, j}$ is the value of the $j^{\text {th }}$ cluster feature and $\left|S_{i}\right|$ is the total number of features in subset $S_{i}$ for $v_{i, j} \in S_{i}$. $\left|S_{i}\right|=2$ for correlated pairs of cluster features. With the inclusion of correlation of parameters, the feature data vector $\boldsymbol{C}_{p_{x}}$ can be expressed as:

$$
\boldsymbol{C}_{p_{x}}=<\tau, v_{1}, \cdots, v_{q}, \varsigma_{1}, \cdots, \varsigma_{\mathrm{K}}>
$$

where integer $q$ is the total number of cluster features, integer $\kappa$ is the total number of correlated feature subsets, and integer $\tau$ is an offset parameter. To compute the growth parameters, the elements of feature data vector $\boldsymbol{C}_{p_{x}}$ are then weighted with a $\mu \times m$ dimensional coefficient matrix $\boldsymbol{A}=\left[a_{i j}\right]$. The relation between the features and the parameters can be stated for a patient $p_{x}$ as:

$$
\boldsymbol{K}_{p_{x}}=\boldsymbol{A} \cdot \boldsymbol{C}_{p_{x}}
$$

where the vector $K_{p_{x}}=<k_{1}, k_{2}, \cdots k_{\mu}>$ for $\mu$ number of tumor growth parameters. Weight coefficients matrix, represented as $A$ in Eq. (3), is computed using the cluster features extracted from H\&E slides. For this purpose, we define vector $\widetilde{K}^{\mathrm{i}}$ of $n$ elements corresponding to different values of the $i^{\text {th }}$ tumor growth parameter for $n$ number of $\mathrm{H} \& \mathrm{E}$ slides as follows:

$$
\widetilde{K}^{i}=\left(\tilde{k}_{i, p 1}, \tilde{k}_{i, p 2}, \cdots, \tilde{k}_{i, p n}\right)
$$

where $\tilde{k}_{i, p x}$ is the value of the $i^{t h}$ parameter for patient $p_{x}$. The set of equations to calculate $\widetilde{K}^{i}$ is:

$$
\widetilde{K}^{i}=A^{i} \cdot\left[\begin{array}{c}
T \\
C_{R}
\end{array}\right]
$$

where $T$ is a single row of $n$ elements of the offset constant $\tau$, and $A^{i}$ is the $i^{\text {th }}$ row of matrix $A$. In Eq. (5), $C_{R}$, the reduced form of matrix $C$, can be formulated as:

$$
C_{R}=\left[v_{i j}\right]
$$

where the constant coefficient $\tau$ is excluded from matrix $\boldsymbol{C}$ to form the $(m-1) \times n$ dimensional matrix $\boldsymbol{C}_{R}$.

Using Eq. (5), we find out each row vector $A^{i}$ of matrix $\boldsymbol{A}$. Plugging vector $A^{i}$ into Eq. 3 , we compute the tumor growth parameters. The explicit formulation to compute the $i^{\text {th }}$ tumor growth parameter $k_{i}$ for patient $p_{x}$ can be represented as:

$$
k_{i}=\tau \cdot a_{i, 1}+\sum_{s=1}^{q+\kappa} \begin{cases}a_{i,(s+1)} \cdot v_{s} & \text { if } s \leq q \\ a_{i,(s+1)} \cdot \zeta_{(s-q)} & \text { otherwise }\end{cases}
$$

where $a_{i j}$ is the $j^{\text {th }}$ coefficient of the row vector $A^{i}$. In our $P R e P-C$ model, we obtain an over-determined system of equations. The artificial intelligence based computation techniques from our previous works [14], [15] is adapted to compute growth parameters of exponential-linear model.

\section{Methodology}

\section{A. H\&E Staining and Preclinical Study}

The research protocol was approved by the Institutional Animal Care and Use Committee (IACUC) to generate patient-derived tumor xenograft models (PDX) in MSKCC. NOD scid gamma (NSG) 4-6 week-old male mice obtained from Jackson Laboratory were used. For tumor implantation process, mice were anesthetized by inhalation with an isoflurane vaporizer. Patient-derived primary tumor cells obtained from kidney cancer patients were cut into small $3 \times 3 \times 3 \mathrm{~mm}^{3}$ fragments and implanted in the flanks of mice subcutaneously. While the mice were still anesthetized carprofen was administered by intraperitoneal injection within 24 hours after surgery.

Tumor growth was monitored twice per week for 30 days. Tumor volume was calculated with the formula: $0.5 \cdot \mathrm{L}$. $W^{2}$ when the length $L$ and width $W$ of tumor mass is measured by caliper. Tumor growth curves are generated using GraphPad Prism software. Tumor mass is excised and cut into $3 \times 3 \times 3 \mathrm{~mm}^{3}$ fragments and implantation process is repeated for more mice when the volume of tumor reaches $1500 \mathrm{~mm}^{3}$. Mice were euthanized if the length $L$ of tumor size measured with caliper reached $2 \mathrm{~cm}$ in length. The experiments have ended approximately in one-month period for each mouse. The tissue samples are collected and fixed in $10 \%$ formalin for histopathological analysis at the end of the experiments. Using microtome $5 \mu \mathrm{m}$ tumor sections were cut, and baked to use for standard H\&E staining.

\section{B. Nuclei Clusters}

Cluster analysis methods organize a collection of unlabelled data set into meaningful groups based on their proximity [8], [9]. For example, two main methods are defined as partitioning and hierarchical clustering, where an optimization rule applied to define clusters for the former type and a recursive approach which results in dendograms is introduced for the latter [7]. K-means clustering defines closeness as the metric for similarity to group data sets into clusters. With $k$-means, the similarity of data set is defined as their closeness, whereas the dissimilarity is determined as the separation of cluster centers in an Euclidian plane [16]. $K$-means aims to determine the best number of clusters for a 
given data set and to divide the data points into the corresponding $K$ groups. The data can be grouped by minimizing the total squared distances between data points and centroids (i.e. geometric mean of clusters) inside a cluster and by maximizing the separation between different clusters [8], [17], [16]. Several algorithms are reported in the literature for computation of the best number of clusters [6], [9], [18], [19]. Jain et al. [9] presents various approaches for clustering including evolutionary approaches, artificial neural networks, and search based approaches to determine the number of clusters and corresponding groups. Liu et al. [6], Murthy et al. [18], and Bandyopadhyay et al. [19] implement genetic algorithm to identify the best structure for clustering. Ray et al. [17] propose a validity measure to draw best structure of clusters based on sum of the distances between cluster centers and data points inside all clusters (i.e. intra distance) and the minimum of the distance between cluster centroids (i.e. inter distance). In this paper, we compute the best number for clusters and their corresponding centroids using our artificial intelligence based techniques developed in our Bio-Inspired Computation Laboratory at the City College of New York [14], [15], [20], [21].

In our process, after selecting $\mathrm{k}$ as the number of clusters, the sets of nuclei belonging to the same clusters are determined. The centroid (i.e. geometric mean) of each cluster is computed as the nucleus with the smallest total distance to the other nuclei in the same cluster. The centroid nuclei set consists of centroids $\varsigma_{i}$ of the clusters $\chi_{i}$ for integer $i \in[1, k]$ and can be computed as:

$$
\varsigma_{i}=\operatorname{argmin}_{x \in \chi_{i}} \sum_{j=1}^{\left|\chi_{i}\right|}\left\|x-v_{j}\right\|^{2}
$$

where the nucleus $v_{j} \in \chi_{i}$ and $\left|\chi_{i}\right|$ is the dimension of cluster $\chi_{i}$. Using the centroids of $\mathrm{k}$ number of clusters and the remaining $n_{i}$ nuclei in Euclidian space where $n_{i}=\left|\chi_{i}\right|-1$, we compute average of intra distances between the centroids and all other nuclei of the same clusters:

$$
\overline{d_{l n}}=\frac{1}{k} \cdot \sum_{i=1}^{k}\left(\frac{1}{n_{i}} \cdot \sum_{v_{j} \in \chi_{i}}\left\|S_{i}-v_{j}\right\|^{2}\right)
$$

and the minimum inter distance between nuclei cluster centroids:

$$
d_{o, \min }=\min \left\|\varsigma_{i}-\varsigma_{j}\right\|^{2}
$$

where integer $i \in[1, k)$ and integer $j \in(i, k]$ identify the compactness and separation of clusters, respectively. The minimum of the distances between centroids (i.e. inter distances) is taken into account to ensure that an acceptable amount of separation is provided among all clusters. We use the ratio of $\overline{d_{l n}} / d_{o, \min }$ as the metric to compute the best number of clusters.

In our study, a digitized H\&E sample slide is processed to identify the nuclei positions which are considered as distinct nodes in the Euclidian space. The nuclei of pathology slides are divided into clusters with the corresponding polygonal regions around their location. In Fig. 1a, the digitized H\&E slide obtained from Sample 51008-R is presented. As the slides are composed of hundreds of nuclei, we select a region (yellow box in Fig. 1a) to focus on the slide details. As shown in Fig. 1b, the best value for the number of clusters is calculated as five for Sample 51008-R. The region of clustering diagram inside the highlighted box in Fig. 1b corresponds to the selected region in Fig. 1a.

\section{Cluster Features}

The H\&E pathology slides can be digitized to extract features based on the positions of the nuclei. Doyle et al. [22] apply textural and graphical feature assignment techniques (e.g. Voronoi tessellation, Delaunay triangulation, and minimum spanning tree, etc.) to analyze the characteristics of the digitized H\&E stained slides. Gurcan et al. [5] list feature extraction methodologies which are used to support pathological studies. Orlov et al. [23] defines multiple features extracted from raw medical images and their various transforms.

In our approach, the spatial distribution of nuclei of kidney cancer tissue samples is analyzed to define the descriptors of images of $H \& E$ pathology slides. We analyze the nuclei clusters obtained from $\mathrm{H} \& \mathrm{E}$ slides using $k$-means clustering approach to extract quantitative results representing cluster characteristic for each slide.

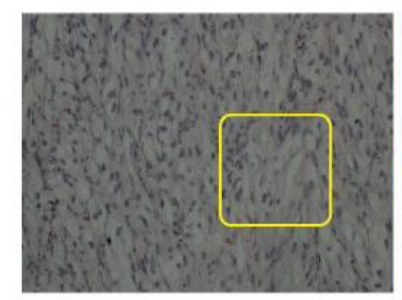

(a) H\&E Slide of Sample 51008-R Tumorous tissue components on H\&E Slide are visualized under light microscope with $20 \mathrm{x}$ magnification. Cell nuclei are stained in blue color by hematoxylin and cytoplasm and connective tissues are stained in pin $^{4}$ color by eosin

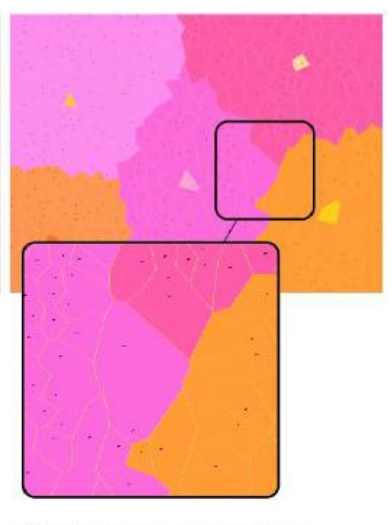

(b) Clusters corresponding to Sample 51008-R
Fig. 1. H\&E stained slide and computed nuclei clusters for sample 51008-R.

TABLE I: MEASUREMENTS FROM SAMPLE H\&E SLIDES

\begin{tabular}{lcccc}
\hline \hline Cluster Features & Sample & Sample & Sample & Sample \\
& $51010 \mathrm{~L}$ & $51008 \mathrm{R}$ & $37385 \mathrm{R}$ & 50052 \\
\hline Number of Clusters & 4 & 5 & 5 & 6 \\
Min Inter Distance & 510.304 & 479.335 & 483.889 & 431.269 \\
Min Cluster Area & 269 & 178 & 194 & 177 \\
Max Cluster Area & 355 & 287 & 299 & 244 \\
Mean Cluster Diameter & 731.018 & 687.461 & 720.408 & 590.096 \\
Min Number of Nodes & 124 & 79 & 94 & 96 \\
Max Number of Nodes & 157 & 128 & 161 & 136 \\
Intra Distance Mean & 216.101 & 199.131 & 194.54 & 175.418 \\
Intra Distance Std. Dev. & 84.947 & 83.512 & 85.129 & 70.395 \\
Intra Distance Skewness & 0.037 & 0.179 & 0.373 & -0.084 \\
\hline \hline
\end{tabular}

The extracted features include the area of clusters, the total number of nuclei in clusters, cluster diameter measurements (i.e. the distance between the most distant two nuclei), statistical measurements of intra distances (i.e. average, standard deviation and skewness of distances), and the minimum distance between cluster centroids. The feature data vector used in our PReP-C model is constructed using 10 cluster descriptors of each H\&E sample as listed in Table I.

In our study, each tumorous tissue sample initially obtained from kidney cancer patients are implanted into 
flanks of multiple mice to increase the variety of samples labelled with different ID numbers. In Table I, the cluster features are listed with the results we obtained for four of the H\&E slide samples, namely 51010-L, 51008-R, 37385-R, and 50052. As can be observed from Table I, the calculated feature values for these slides show that the number of clusters is inversely proportional to several other features (e.g., the cluster size and the number of nuclei).

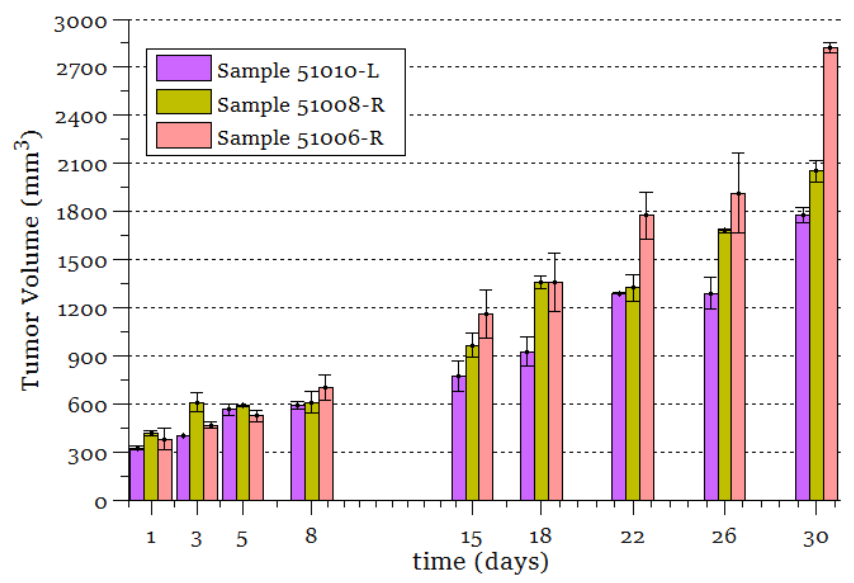

(a). Tumor growth for experiment schedule $\mathrm{A}$.

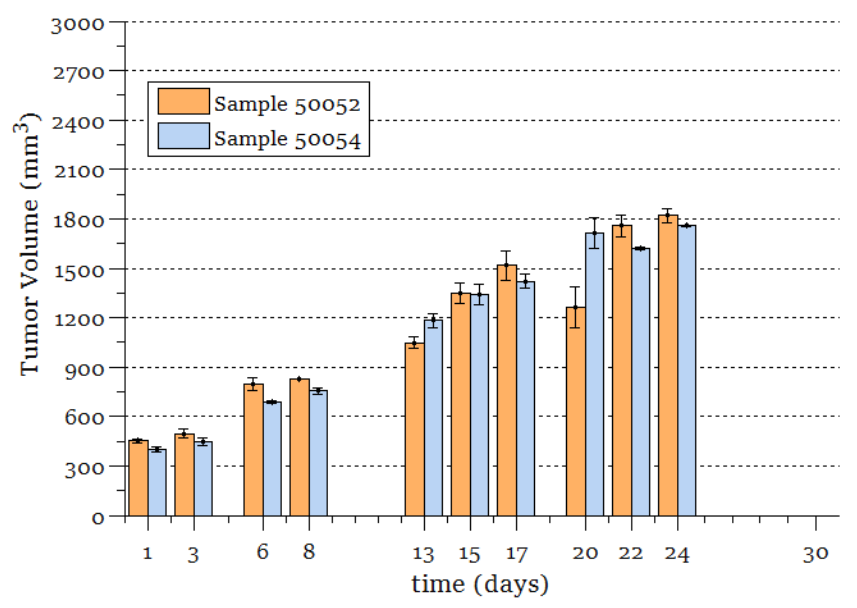

(b). Tumor growth for experiment schedule B.

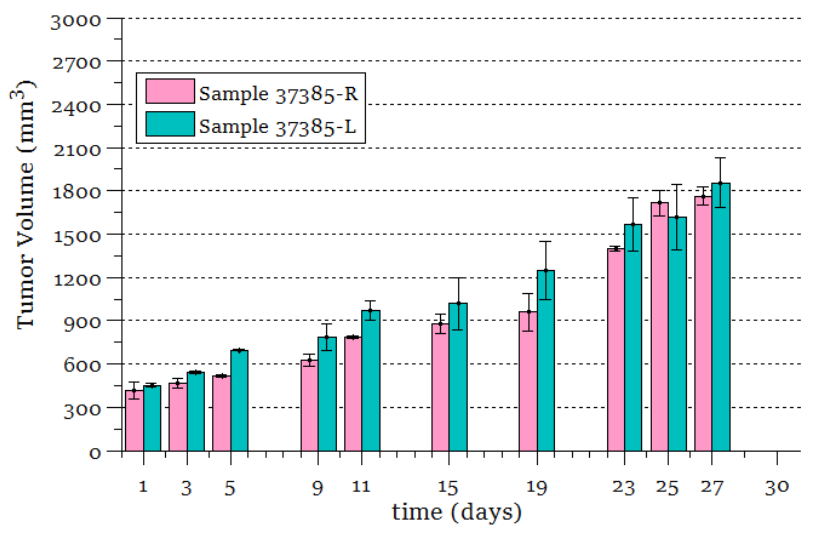

(c) Tumor growth for experiment Schedule C.

Fig. 2. Preclinical tumor volume measurements and error ranges of PReP-C results for 7 sample H\&E slides.

\section{RESUlTS AND DisCUSSION}

In this paper, we focus on the linear phase of tumor growth as the preclinical stage of the study was conducted after the tumor volume has reached $400 \mathrm{~mm}^{3}$ and the tumor mass has already started to proliferate at a linear rate. The linear tumor growth parameter $\lambda_{1}$ of exponential-linear tumor growth model [1] is computed with our PReP-C model with the feature data vectors generated using the information obtained from $14 \mathrm{H} \& \mathrm{E}$ slide samples.

Volume of tumor mass in mice was measured at certain days during the one-month period in preclinical phase of our study. We evaluate the performance of our PReP-C model using the leave-one-out-cross-validation (LOOCV) technique [24]. In Fig. 2, we represent the preclinical tumor volume measurements with the LOOCV error rates corresponding to $P \operatorname{ReP}-\mathrm{C}$ results for 7 of the 14 sample slides. The results shown are grouped based on the three different daily experimental schedules. In Fig. 2a, the pre-clinical measurements and the results computed with our PReP-C model for the H\&E slides of 51010-L, 51008-R, and 51006-R are shown. The measured and computed volumes for Sample Slides of 50052 and 50054 are given in Fig. 2b, and for Slides 37385-R and 37385-L in Fig. 2c. The difference between preclinical volume measurements and volume results computed with $P R e P-C$ model is represented with the error bars on top of each volume bar, where the magnitude of bars corresponds to the difference.

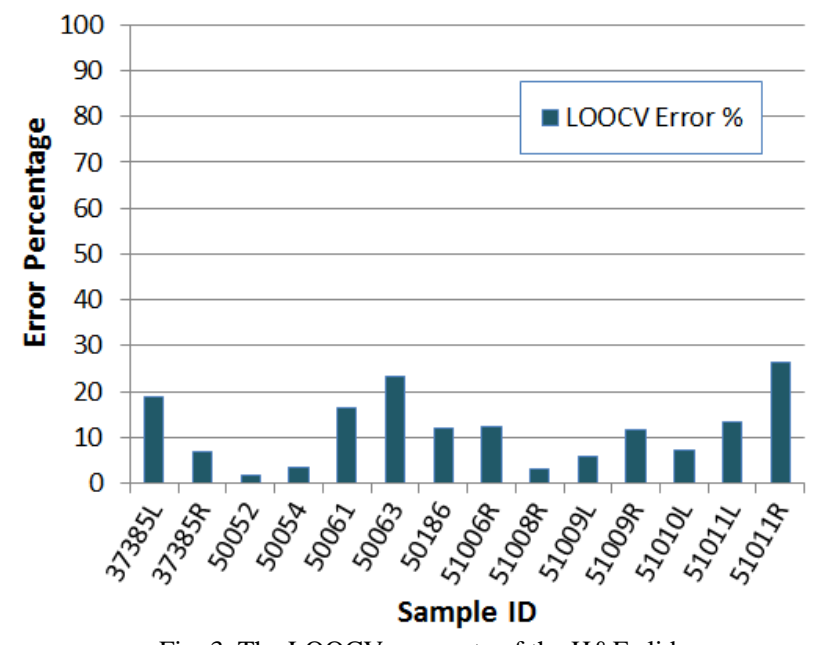

Fig. 3. The LOOCV error rate of the H\&E slides.

As illustrated in Fig. 2a, the results computed with our PReP-C model for the H\&E slide samples 51010-L, 51008-R, and 51006-R are close to the preclinical measurements, even though the tumor volumes are significantly different for each sample. A similar consistency between the measured and computed results can be observed for the samples shown in Figs. $2 b$ and $2 c$ although the experiments were conducted in different cycles. The LOOCV error rates for the measured and computed tumor volume results for 14 sample slides are presented in Fig. 3. Despite the variations on pre-clinical tumor volume measurements and the experiment schedules, the average error rate for all samples is calculated as $11.6 \%$.

The goal of our $P R e P-C$ model is to study the construction of personalized mathematical models for tumor progression. PReP-C was able to generate tumor growth curves with a relatively small error based on the features extracted from the $14 \mathrm{H} \& \mathrm{E}$ slides of kidney cancer patients. We are planning to extent our study on personalized mathematical models using information from a larger set of pre-clinical experiments including the measurements for tumor shrinkage. 


\section{REFERENCES}

[1] M. Simeoni, P. Magni, C. Cammia et al., "Predictive pharmacokinetic pharmacodynamic modeling of tumor growth kinetics in xenograft models after administration of anticancer agents," Cancer Research, vol. 64, pp. 1094-1101, 2004.

[2] E. Ganic, S. Gundry, J. Zou, and M. U. Uyar, "Evaluation of anti-cancer therapy using in silico analysis of treatments for HER2+ breast cancer.," in Proc. the Symposium on Medical Measurements and Applications, 2014, pp. 211-216, Lisbon, Portugal.

[3] G. Koch, A. Walz, G. Lahu et al., "Modeling of tumor growth and anticancer effects of combination therapy," Journal of Pharmacokinetcs and Pharmacodynamics, vol. 36, pp. 179-197, 2009.

[4] A. Saribudak, E. Ganic, J. Zou, S. Gundry, and M. U. Uyar, "Toward genomic based personalized mathematical models for breast cancer tumor growth," in Proc. the 14th Int'l. Conference on BioInformatics and BioEngineering (BIBE), 2014, pp. 115-119.

[5] M. N. Gurcan, L. E. Boucheron, A. Can, A. Madabhushi, N. M. Rajpoot, and B. Yener, "Histopathological image analysis: A review," IEEE Reviews in Biomedical Eng, vol. 2, pp. 147-171, 2009.

[6] C. Liu, T. Hu, Y. Ge, and H. Xiong, "Which distance metric is right: An evolutionary k-means view," SDM, pp 907-918, 2012.

[7] M. Filippone, F. Camastra, F. Masulli and S. Rovetta, "A survey of kernel and spectral methods for clustering," Pattern Recognition, vol. 41, no. 1, pp. 176-190, 2008.

[8] D. Soria, J. M. Garibaldi, F. Ambrogi, A. R. Green, D. Powe, E. Rakha, R. Douglas Macmillan, R. W. Blamey, G. Ball, P. J. Lisboa et al., “A methodology to identify consensus classes from clustering algorithms applied to immunohistochemical data from breast cancer patients," Computers in biology and medicine, vol. 40, no. 3, pp. 318-330, 2010.

[9] A. K. Jain, M. N. Murty, and P. J. Flynn, "Data clustering: a review," ACM computing surveys (CSUR), vol. 31, no. 3, pp. 264-323, 1999.

[10] M. Rocchetti, M. Simeoni, E. Pesenti et al., "Predicting the active doses in humans from animal studies: A novel approach in oncology," European Journal of Cancer, vol. 43, no. 12, pp. 1862 -1868, 2007.

[11] J. Harrold and R. Parker, "Clinically relevant cancer chemotherapy dose scheduling via mixed-integer optimization," Computers and Chemical Engineering, vol. 33, no. 12, pp. 2042-2054, 2009.

[12] R. Edgar, M. Domrachev, and A. E. Lash, "Gene expression omnibus: Ncbi gene expression and hybridization array data repository," Nucleic acids research, vol. 30, no. 1, pp. 207-210, 2002.

[13] NBIA - National Biomedical Imaging Archive. National Institutes of Health. [Online]. Available: https://imaging.nci.nih.gov/ncia.

[14] J. Kusyk, C. Sahin, M. Uyar, E. Urrea, and S. Gundry, "Self organization of nodes in mobile ad hoc networks using evolutionary games and genetic algorithms," Journals of Adv. Res., vol. 2, no. 3, pp. $253-264,2011$.

[15] C. S. Sahin, S. Gundry, and M. U. Uyar, "Markov chain analysis of selforganizing mobile nodes," Journal of Intelligent and Robotic Systems, vol. 67, pp. 133-153, 2012.

[16] D. L. Davies and D. W. Bouldin, "A cluster separation measure," IEEE Transactions on Pattern Analysis and Machine Intelligence, vol. 2, pp. 224-227, 1979.

[17] S. Ray and R. H. Turi, "Determination of number of clusters in $\mathrm{k}$-means clustering and application in colour image segmentation," in Proc. the 4th International Conference on Advances in Pattern Recognition and Digital Techniques, pp. 137-143, 1999.

[18] C. A. Murthy and N. Chowdhury, "In search of optimal clusters using genetic algorithms," Pattern Recognition Letters, vol. 17, no. 8, pp 825-832, 1996.

[19] S. Bandyopadhyay and S. S. Gaps, "A clustering method using a new point symmetry-based distance measure," Pattern Recognition, vol. 40 no. 12, pp. 3430-3451, 2007.

[20] S. Gundry, J. Zou, E. Urrea, C. Sahin, J. Kusyk, and M. Uyar, "Analysis of emergent behavior for ga-based topology control mechanism for self-spreading nodes in MANETs," Advances in Intelligent Modelling and Simulation, pp. 155-183. Springer Berlin Heidelberg, 2012.
[21] J. Zou, S. Gundry, J. Kusyk, M. U. Uyar, and C. S. Sahin, "3D genetic algorithms for underwater sensor networks," International' Journal of Ad Hoc and Ubiquitous Computing, vol. 13, no. 1, pp. 10-22, 2013.

[22] S. Doyle, S. Agner, A. Madabhushi, M. Feldman, and J. Tomaszewski, "Automated grading of breast cancer histopathology using spectral clustering with textural and architectural image features," in Proc. 5th IEEE Signal Processing Society (SPS) and IEEE Engineering in Medicine and Biology Society (EMBS),2008, pp. 496-499.

[23] N. Orlov, L. Shamir, T. Macura, J. Johnston, D. M. Eckley, and I. G. Goldberg, "Wnd-charm: Multi-purpose image classification using compound image transforms," Pattern Recognition Letters, vol. 29, no. 11, pp. 1684-1693, 2008.

[24] M. S. Arlot, A. Celisse et al., "A survey of cross-validation procedures for model selection,” Statistics surveys, vol. 4, pp. 40-79, 2010.

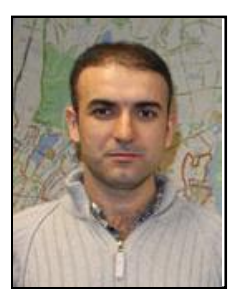

Aydin Saribudak received his bachelor in science degree from Electrical and Electronics Engineering Department of Middle East Technical University, Turkey. He is currently a Ph.D. candidate at the City College of the City University of New York. His research interests include biologically inspired computation algorithms, artificial intelligence, and development of personalized mathematical models for tumor growth and anti-cancer therapy.

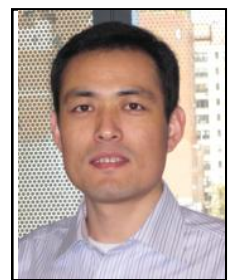

Yiyu Dong obtained his Ph.D. degree from Peking University in China, and completed his $\mathrm{Ph}$. D. dissertation at Yale University in the United States as a Monsanto Fellowship awardee. In 2010, he joined Dr. Hsieh's Laboratory as a research associate in human oncology and pathogenesis program in Memorial Sloan Kettering Cancer Center. Dr. Dong's research interests focus on translational science of human oncology, especially chemotherapy and immunotherapy using pre-clinical models.

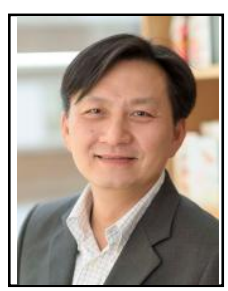

James J. Hsieh obtained his Ph.D. degree from Johns Hopkins Medical School. Dr. Hsieh joined medical and oncology training program in Washington University and Dana Farber Cancer Institute. He joined the late Dr. Korsmeyer's Laboratory As an HHMI physician-scientist fellowship awardee. as an NCI K01 Howard Temin Awardee, he joined the faculty at Washington University in 2004. Since 2010 he has been a physician scientist in Memorial Sloan Kettering Cancer Center. Dr. Hsieh is an associate member of Human Oncology and Pathogenesis Program.

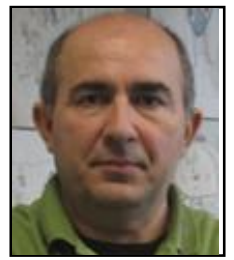

M. Umit Uyar is with Electrical Engineering Department of the City College of New York and the Computer Science Department of the Graduate Center of the City University of New York. His research includes applications of bio-inspired computation techniques to personalized anti-cancer therapy and decision support systems. Prior to joining academia, he was a distinguished member of Technical Staff at AT\&T Bell Labs. Dr. M. Umit Uyar has a B.S. degree from Istanbul Technical University and M.S. and Ph.D. degrees from Cornell University, Ithaca, NY, all in electrical engineering. He is an IEEE Fellow and holds six U.S. patents. 